\title{
SOME APPLICATIONS OF THE INEQUALITY OF ARITHMETIC AND GEOMETRIC MEANS TO POLYNOMIAL EQUATIONS
}

HERBERT S. WILF

The purpose of this note is to point out a simple generalization of the inequality

$$
\left(z_{1} z_{2} \cdots z_{n}\right)^{1 / n} \leqq \frac{1}{n}\left(z_{1}+\cdots+z_{n}\right)
$$

of arithmetic and geometric means, which will hold when the arguments of the complex numbers $z_{1}, \cdots, z_{n}$ are suitably restricted. We shall apply the resulting inequality to the roots of polynomial equations, obtaining first a quantitative form of the Gauss-Lucas theorem, and then some relationships between the coefficients of a polynomial and the size of a sector containing its roots.

1. The inequality. The basic result is

Theorem 1. Suppose

$$
\left|\arg z_{i}\right| \leqq \psi<\frac{\pi}{2}, \quad i=1,2, \cdots, n .
$$

Then

$$
\left|z_{1} z_{2} \cdots z_{n}\right|^{1 / n}<(\sec \psi) \frac{1}{n}\left|z_{1}+z_{2}+\cdots+z_{n}\right|
$$

unless $n$ is even and $z_{1}=\cdots=z_{n / 2}=\bar{z}_{(n / 2)+1}=\cdots=\bar{z}_{n}=r e^{i \psi}$, in which case equality holds.

Proof. We have

$$
\begin{aligned}
\left|z_{1}+z_{2}+\cdots+z_{n}\right| \geqq & \left|\operatorname{Re}\left(z_{1}+\cdots+z_{n}\right)\right| \\
= & \left(\left|z_{1}\right| \cos \phi_{1}+\left|z_{2}\right| \cos \phi_{2}+\cdots\right. \\
& \left.+\left|z_{n}\right| \cos \phi_{n}\right) \\
\geqq & (\cos \psi)\left(\left|z_{1}\right|+\cdots+\left|z_{n}\right|\right) \\
\geqq & n \cos \psi\left(\left|z_{1}\right|\left|z_{2}\right| \cdots\left|z_{n}\right|\right)^{1 / n}
\end{aligned}
$$

Received by the editors March 2, 1962. 
as claimed. All signs of equality hold only when
(a) $\operatorname{Im}\left(z_{1}+\cdots+z_{n}\right)=0$
(b) $\cos \phi_{i}=\cos \psi \quad(i=1,2, \cdots, n)$
(c) $\left|z_{1}\right|=\left|z_{2}\right|=\cdots=\left|z_{n}\right|$

which imply the configuration stated in the theorem. For odd $n$ the constant sec $\psi$ is only asymptotically best possible.

2. Application to polynomials. Let

$$
P(z)=a_{0}+a_{1} z+\cdots+a_{n} z^{n}=a_{n}\left(z-z_{1}\right) \cdots\left(z-z_{n}\right)
$$

be given and let $K$ denote the convex hull of the zeros $z_{1}, \cdots, z_{n}$ of $P(z)$. Let $z$ be outside $K$, and suppose that, from $z, K$ subtends an angle $2 \psi$. Then the spread in the arguments of the numbers

$$
\frac{1}{z-z_{1}}, \cdots, \frac{1}{z-z_{n}}
$$

is at most $2 \psi$, and from Theorem 1 ,

$$
\left|\frac{1}{\left(z-z_{1}\right)} \cdots \frac{1}{\left(z-z_{n}\right)}\right|^{1 / n} \leqq(\sec \psi) \frac{1}{n}\left|\sum_{\nu=1}^{n} \frac{1}{z-z_{\nu}}\right| .
$$

But this is just the assertion that

$$
\left|\frac{a_{n}}{P(z)}\right|^{1 / n} \leqq \frac{\sec \psi}{n}\left|\frac{P^{\prime}(z)}{P(z)}\right|,
$$

and we have proved

THEOREM 2. If $z$ is a point from which the convex hull of the zeros of the polynomial $P(z)$ of degree $n$ subtends an angle $2 \psi<\pi$, then

$$
\left|P^{\prime}(z)\right| \geqq n\left|a_{n}\right|^{1 / n}(\cos \psi)|P(z)|^{1-(1 / n)} \text {. }
$$

COROLlary 1. The zeros of $P^{\prime}(z)$ lie in the convex hull of the zeros of $P(z)$ (Gauss-Lucas).

COROLlaRy 2. If the zeros of $P(z)$ lie in the unit circle, then we have for $|z|>1$,

$$
\left|P^{\prime}(z)\right| \geqq \frac{n\left|a_{n}\right|^{1 / n}}{\sqrt{ }\left(1-\frac{1}{|z|^{2}}\right)}|P(z)|^{1-(1 / n)}
$$


TheOREM 3. The zeros of the polynomial

$$
P(z)=a_{0}+a_{1} z+\cdots+a_{n} z^{n},
$$

are not contained in any sector of central angle less than

$$
2 \cos ^{-1}\left\{\left|\frac{a_{n-1}}{n a_{n}}\right| \min _{0 \leqq k \leqq n-1}\left|\frac{a_{n}}{a_{k}}\left(\begin{array}{l}
n \\
k
\end{array}\right)\right|^{1 / n-k}\right\} .
$$

Proof. Suppose the zeros of $P(z)$ lie in a sector of angle $2 \psi<\pi$. From Theorem 1,

$$
\left|\frac{a_{0}}{a_{n}}\right|^{1 / n} \leqq \frac{\sec \psi}{n}\left|\frac{a_{n-1}}{a_{n}}\right|,
$$

or

$$
\sec \psi \geqq n\left|a_{n}\right|^{1-(1 / n)}\left|a_{0}\right|^{1 / n}\left|a_{n-1}\right|^{-1} .
$$

Applying this result to

$$
P^{(k)}(z)=\sum_{\nu=0}^{n-k} \frac{(\nu+k) !}{\nu !} a_{\nu+k} z^{\nu}
$$

which, by Corollary 1 also satisfies the hypotheses, we find

$$
\sec \psi \geqq\left|\frac{n a_{n}}{a_{n-1}}\right|\left|\frac{a_{n}}{a_{k}}\left(\begin{array}{l}
n \\
k
\end{array}\right)\right|^{-1 /(n-k)} \quad(k=0,1, \cdots, n-1),
$$

and the result follows.

TheOREM 4. Under the hypotheses of Theorem 2, let $\rho$ denote the distance from $z$ to the center of gravity of the zeros of $P(z)$. Then

$$
|P(z)| \leqq\left|a_{n}\right|(\rho \sec \psi)^{n} \text {. }
$$

Proof. Apply Theorem 1 to the numbers $z-z_{1}, \cdots, z-z_{n}$.

UNIVERSITY OF ILLINOIS 